\title{
Preparação e caracterização de poliuretanos contendo diferentes quantidades de óleo de baru
}

\section{Preparation and characterization of polyurethane with different quantities of baru oil}

\author{
Elizabeth Luiza de Almeida', Gilberto Alessandre Soares Goulart ${ }^{2}$, Salvador Claro Neto ${ }^{3}$, \\ Gilberto Orivaldo Chierice ${ }^{3}$ e Adriano Buzutti de Siqueira1* \\ ${ }^{1}$ Laboratório de Estudos de Materiais - LEMat, Universidade Federal de Mato Grosso - UFMT, \\ Cuiabá, MT, Brasil \\ ${ }^{2}$ Escola de Engenharia de Alimentos - EA, Universidade Federal de Goiás - UFG, Goiânia, GO, Brasil \\ ${ }^{3}$ Instituto de Química de São Carlos - IQSC, Universidade de São Paulo - USP, São Carlos, SP, Brasil \\ *buzutti7@hotmail.com
}

\begin{abstract}
Resumo
O óleo de baru $(\mathrm{OB})$ é uma matéria-prima renovável proveniente do cerrado brasileiro que possui elevado percentual de insaturação. O objetivo principal do trabalho foi o estudo das espumas de poliuretano com diferentes quantidade de óleo de baru saponificado. Os espectros de FTIR mostraram bandas representativas do poliol, óleo de baru e do pré-polímero. As curvas TG-DSC nas atmosferas de ar e $\mathrm{N}_{2}$ apresentaram perfis diferentes, devido a formação de produtos intermediários de oxidação dos materiais na atmosfera de ar. A quantidade de energia liberada pela DSC e as perdas de massa observadas pelas curvas TG indicaram saturação do óleo de baru na quantidade de $24,00 \%$ (m/m). Os gases liberados analisados por TG-DSC acoplado ao FTIR, foram: água, $\mathrm{CO}_{2}, \mathrm{CO}$ e etanol. Com o aumento do óleo de baru, ocorreu maior absorção de água pelo PU, na proporção de $29,00 \%$ ( $\mathrm{m} / \mathrm{m})$ o índice de intumescimento foi de 91,10\%, em 1440 minutos. Assim, pudemos observer a formação do poliuretano com características distintas devido a presença do $\mathrm{OB}$.
\end{abstract}

Palavras-chave: poliuretano, óleo de baru, TG/DSC, FTIR.

\begin{abstract}
The baru oil $(\mathrm{OB})$ is a renewable raw material from the brazilian cerrado, with high unsaturation percentage. The objective of this work was the study of polyurethane foam with different quantities of baru saponified oil. FTIR spectrum showed the bands of polyol, baru oil and prepolymer. The TG-DSC curves in $\mathrm{N}_{2}$ and air indicated different profiles, especially the formation of more stable intermediate products on the oxidation of these materials in air atmosphere. The quantity of heat obtained by DSC curves and the weight loss obtained by TG curve, indicates an OB saturation at $24.00 \%$ (w/w). The study by FTIR of the volatile products released by TG-DSC were water, $\mathrm{CO}_{2}, \mathrm{CO}$ and etanol. With the increase of OB, higher water absorption of PU, in $29.00 \%$ (w/w) of swelling index $91.10 \%$ for 1440 minutes. From these results, we observe the formation of polyurethane with different characteristics because of OB presence.
\end{abstract}

Keywords: polyurethane, baru oil, TG/DSC, FTIR.

\section{Introdução}

Os óleos vegetais, formados principalmente por triglicerídeos, fornecem uma excelente plataforma para síntese de materiais poliméricos. Devido a sua baixa toxicidade, baixo custo de produção e processamento, e por serem geralmente biodegradáveis. Vários tipos de óleos vegetais estão sendo utilizados para síntese de polímeros, tais como: soja, canola, girassol e linhaça, devido as suas excelentes propriedades ${ }^{[1,2]}$.

O baru (Dipteryx alata Vog.), árvore da família Fabaceae, disseminada no Bioma Cerrado, faz parte do grupo das espécies nativas usadas pela população regional como fonte de renda familiar ${ }^{[3]}$. Apresenta frutos do tipo drupa, ovóides, levemente achatados e de coloração marrom, com uma única semente (amêndoa) comestível e comercializada em empórios nos grandes centros, bastante apreciada pela população local. Se prolifera na região do Planalto Central, precisamente no norte de Minas Gerais, Goiás e centro de Mato Grosso, indo até a costa atlântica do Maranhão ${ }^{[4-6]}$.

Em estudo realizado por Takemoto et al. ${ }^{[4]}$, a semente de baru apresentou teores relativamente elevados de lipídios 
$(38,2 \mathrm{~g} / 100 \mathrm{~g})$ de proteínas $(23,9 \mathrm{~g} / 100 \mathrm{~g})$ e de calorias $(502 \mathrm{kcal} / 100 \mathrm{~g})$, além de fibras alimentares $(13,4 \mathrm{~g} / 100 \mathrm{~g})$ e de minerais, como potássio $(827 \mathrm{mg} / 10 \mathrm{~g})$, fósforo $(358 \mathrm{mg} / 100 \mathrm{~g})$ e magnésio $(178 \mathrm{mg} / 100 \mathrm{~g})$. O óleo da semente revelou um elevado grau de insaturação $(81,2 \%)$, conteúdo de $\alpha$-tocoferol $(5,0 \mathrm{mg} / 100 \mathrm{~g})$ e composição em ácidos graxos semelhantes a do óleo de amendoim, destacando-se os ácidos oléicos $(50,4 \%)$ e linoléico $(28,0 \%)$, este considerado essencial, o que favorece seu uso para fins alimentícios e como matéria-prima para as indústrias farmacêutica e oleoquímica.

Poliuretano (PU) é um dos materiais poliméricos mais versáteis, em relação aos seus métodos de processamento e suas propriedades mecânicas, podendo-se obter um plástico rígido cristalino, um elastômero flexível ou um gel viscoelástico ${ }^{[7,8]}$. Podendo ainda ser utilizado como espumas (flexíveis, rígidas e semi-rígidas), revestimentos, adesivos e fibras ${ }^{[9]}$.

O objetivo do trabalho foi preparar e caracterizar os poliuretanos obtidos a partir da mistura do pré-polímero MDI (difenilmetano diisocianato), do poliol de óleo de mamona adicionando óleo de baru em diferentes proporções de massa $(5,00 \%, 15,00 \%, 24,00 \%$ e $29,00 \%)$.

\section{Materiais e Métodos}

Todos os reagentes utilizados são de grau analítico, sendo o $\mathrm{NaOH}$ da marca Synth com grau de pureza $99,9 \%$ e etanol da marca Synth P.A. A obtenção do óleo de Baru bruto (OBB) ocorreu a partir de extração das amêndoas do baru, armazenadas $\mathrm{a}-18^{\circ} \mathrm{C}$, em hexano. O pré-polímero $(\mathrm{PP})$ e o poliol utilizados na síntese foram desenvolvidos pelo Grupo de Química Analítica e Tecnologia de Polímeros-GQATP, USP de São Carlos-SP e produzidos pela indústria Cequil. O pré-polímero é baseado em difenilmetano diisocianato (MDI) e o poliol de óleo de mamona.

\subsection{Preparação dos poliuretanos}

O OBB foi saponificado em balão de fundo redondo, foi adicionado $120,0 \mathrm{~mL}$ de água destilada em mistura contendo $\mathrm{OBB}(20,0 \mathrm{~g}) / \mathrm{NaOH}(4,00 \mathrm{~g}) / \mathrm{CH}_{3} \mathrm{CH}_{2} \mathrm{OH}(40,0 \mathrm{~mL})$. O balão de fundo redondo foi mantido por 2 horas colocado em rotaevaporador da LOGEN Scientific, modelo LSCS-1/52. A mistura foi transferida para béquer, sendo ajustado para valor de $\mathrm{pH}$ igual a 4 com adição de solução aquosa de ácido sulfúrico $30,0 \%(\mathrm{v} / \mathrm{v})^{[10]}$.

A mistura do óleo de baru saponificado (OBS) com o PP e o poliol foi realizado por agitação manual de $18,00 \mathrm{~g} P \mathrm{PP}$ $2,00 \mathrm{~g}$ poliol em respectivos $1,00 \mathrm{~g}(5,00 \%), 3,00 \mathrm{~g}(15,00 \%)$, $5,00 \mathrm{~g}(24,00 \%)$ e $6,00 \mathrm{~g}(29,00 \%)$ de OBS.

\subsection{Métodos de caracterização}

Os espectros de absorção na região do infravermelho com Transformada de Fourier (FTIR) foram obtidos no Espectrofotômetro Perkin Elmer, modelo Spectrum 100, com resolução $4 \mathrm{~cm}^{-1}$, na região compreendida entre $4.000-600 \mathrm{~cm}^{-1}$, utilizando a técnica de reflectância total atenuada (ATR) com cristal de diamante/ZnSe.

As curvas TG-DSC foram obtidas no equipamento TG/DSC-1 da Mettler Toledo. O sistema foi calibrado seguindo as especificações fornecidas pelo fabricante. As curvas foram obtidas em cadinho de $\alpha-\mathrm{Al}_{2} \mathrm{O}_{3}(70 \mu \mathrm{L})$, com massa de amostra de aproximadamente $6 \mathrm{mg}$, razão de aquecimento de $20^{\circ} \mathrm{C} \mathrm{min}^{-1}$, atmosfera de ar seco e $\mathrm{N}_{2}$ com vazão de $60 \mathrm{~mL} \mathrm{~min}^{-1} \mathrm{e}$ intervalo de temperatura de $30-1000^{\circ} \mathrm{C}$.

As curvas TG-DSC e os espectros de infravermelho dos produtos gasosos foram obtidas no equipamento TGA/SDTA 851 da Mettler Toledo acoplado ao sistema de espectrometria infravermelha com transformada de Fourier, modelo Nicolet iS10 FTIR.

Para a determinação do percentual de intumescimento (Ii\%), os PU's foram cortados em pedaços de $1 \mathrm{~cm}^{3}$ e mantidas em dessecador por 7 dias, os testes realizados em triplicata. Os PU's foram submersos em água destilada, e em cada intervalo de tempo (1 $\mathrm{min}, 2 \mathrm{~min}, 5 \mathrm{~min}, 10 \mathrm{~min}$, $15 \mathrm{~min}, 20 \mathrm{~min}, 30 \mathrm{~min}, 60 \mathrm{~min}$ e $1440 \mathrm{~min}$ ), as peças eram cuidadosamente removidas, utilizando-se pinça, sendo retirado o excesso de água com papel toalha e, em seguida, as massas dos PU's eram medidas. Este procedimento foi realizado de acordo com Soares ${ }^{[1]]}$ e Pereira et al. ${ }^{[12]}$.

Foi construído um gráfico de intumescimento gravimétrico, utilizando-se a equação abaixo:

$$
I i \%=\frac{\text { Massa Final }- \text { Massa Inicial } x 100}{\text { Massa Inicial }}
$$

\section{Resultados e Discussão}

O óleo de baru foi submetido à saponificação para a liberação de hidroxilas nas cadeias, além de tornar o óleo miscível, este processo facilita a reação com o PP e o poliol na reação de polimerização. $\mathrm{O}$ aspecto visual dos $\mathrm{PUs}$ com OB indicaram tamanho de poros diferentes, indicando a influência da quantidade de OB no processo de polimerização.

\subsection{Análise térmica do PP, Poliol, OBB e OBS}

\subsubsection{TG-DSC do OBB}

As curvas TG-DSC do OBB mostram a decomposição térmica em 3 (atmosfera $\mathrm{N}_{2}$ ) e 4 (atmosfera de ar) etapas consecutivas, ver Figuras 1 e 2 e Tabelas 1 e 2 . A primeira etapa de decomposição térmica são equivalentes a saída de água adsorvida do ambiente, sendo observado perda de massa inferior a 1\%, indicando ser material não higroscópico. A segunda etapa de decomposição térmica são referentes a saída de compostos orgânicos voláteis de baixa massa molecular e provavelmente a evaporação do ácido gadoléico, presentes no $\mathrm{OBB}^{[4]}$.

Em atmosfera de ar foram observados eventos exotérmicos na terceira etapa de decomposição térmica referentes a oxidação de compostos presentes. A terceira etapa de decomposição térmica, obervada em atmosfera de $\mathrm{N}_{2}$, foi devido a evaporação de compostos com massas molares maiores $\left(\mathrm{C}_{16: 0}, \mathrm{C}_{18: 0}, \mathrm{C}_{18: 1}, \mathrm{C}_{18: 2}, \mathrm{C}_{20: 0}, \mathrm{C}_{22: 0}\right)$, ácido lignocérico e compostos de $\alpha$ e $\gamma$-tocoferol. A formação de produtos intermediários oxidados em ar, diferente da evaporação, inferiu maior temperatura final $\left(\mathrm{T}_{\mathrm{f}}=647^{\circ} \mathrm{C}\right)$ na decomposição térmica do $\mathrm{OBB}$. Os resíduos estáveis formados até $1000^{\circ} \mathrm{C}, 1,15 \%(\operatorname{ar})$ e $1,20 \%\left(\mathrm{~N}_{2}\right)$, são referentes as impurezas (material inorgânico) dos materiais. 

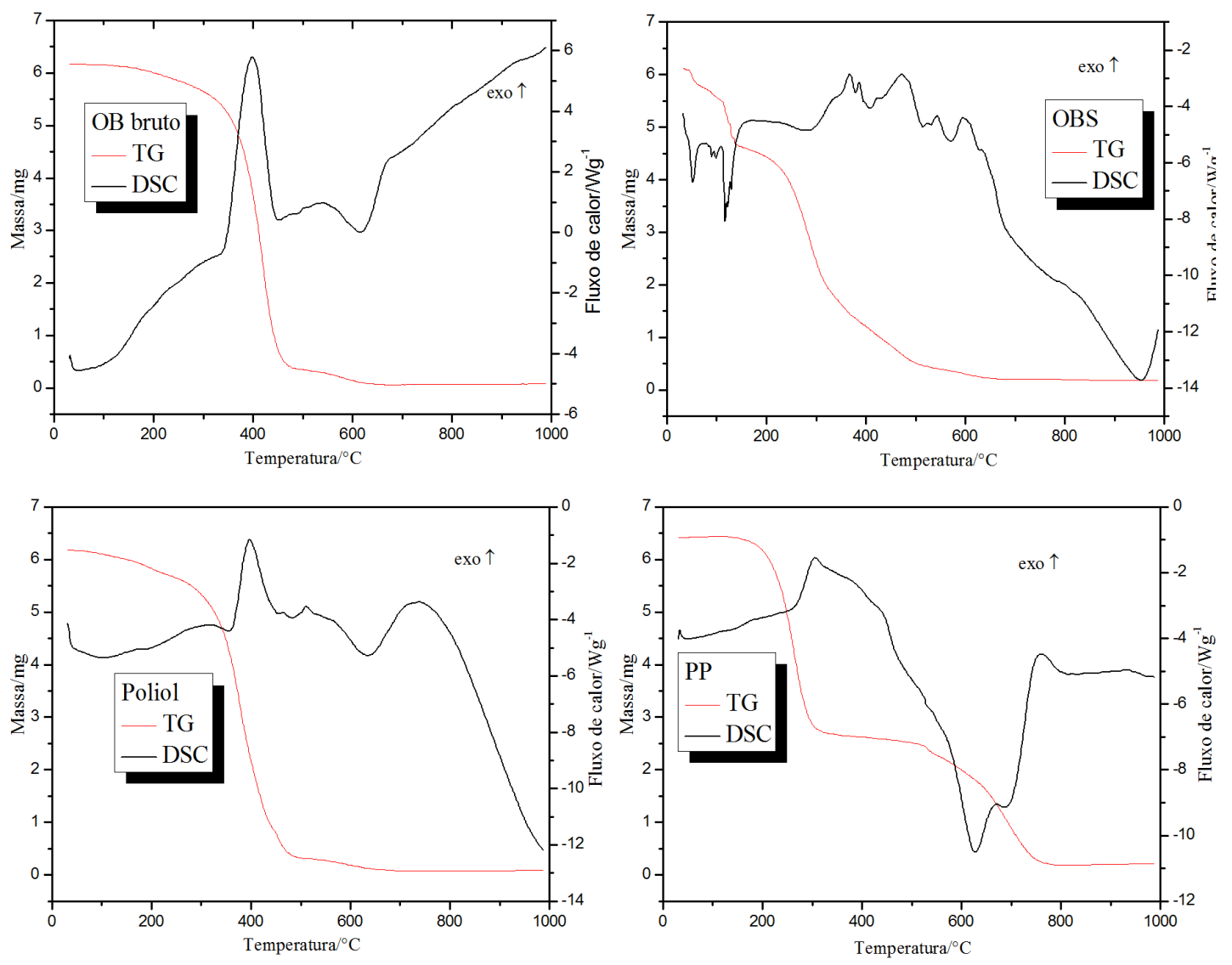

Figura 1. TG-DSC das matérias-primas dos poliuretanos em atmosfera de ar.

Tabela 1. Dados termoanaliticos das matérias-primas dos poliuretanos em atmosfera de ar seco.

\begin{tabular}{|c|c|c|c|c|c|c|c|c|c|c|c|c|}
\hline \multirow{2}{*}{ Amostra } & \multicolumn{3}{|c|}{ Primeira etapa } & \multicolumn{3}{|c|}{ Segunda etapa } & \multicolumn{3}{|c|}{ Terceira etapa } & \multicolumn{3}{|c|}{ Quarta etapa } \\
\hline & $\mathbf{T}^{\circ} \mathbf{C}$ & $\Delta \mathrm{m} \%$ & Pico/ $/{ }^{\circ} \mathrm{C}$ & $\mathbf{T}^{\circ} \mathrm{C}$ & $\Delta \mathrm{m} \%$ & Pico $/{ }^{\circ} \mathrm{C}$ & $\mathrm{T}^{\circ} \mathrm{C}$ & $\Delta \mathbf{m} \%$ & Pico $/{ }^{\circ} \mathrm{C}$ & $\mathbf{T}^{\circ} \mathbf{C}$ & $\Delta \mathrm{m} \%$ & Pico/ $/{ }^{\circ} \mathrm{C}$ \\
\hline OB & $67-140$ & 0,69 & - & $140-250$ & 4,37 & $225(\uparrow)$ & $250-478$ & 88,53 & $338(\uparrow)$ & $478-647$ & 5,14 & $616(\uparrow)$ \\
\hline OBS & $36-85$ & 6,88 & $52(\downarrow)$ & $85-138$ & 16,52 & $\begin{array}{c}90,99 \\
117,123 \\
\text { e } 128(\downarrow) \\
\end{array}$ & $138-502$ & 68,20 & $\begin{array}{c}369, \\
386,423, \\
474(\uparrow)\end{array}$ & $502-650$ & 4,57 & $\begin{array}{c}524, \\
543,595, \\
634(\uparrow)\end{array}$ \\
\hline Poliol & $47-174$ & 3,93 & $105(\downarrow)$ & $174-489$ & 90,35 & $\begin{array}{c}298,397, \\
467(\downarrow)\end{array}$ & $489-685$ & 4,18 & $635(\downarrow)$ & - & - & - \\
\hline $\mathbf{P P}$ & $135-339$ & 58,32 & $306(\uparrow)$ & $339-784$ & 38,62 & $\begin{array}{c}628, \\
690(\downarrow) \\
\end{array}$ & - & - & - & - & - & - \\
\hline
\end{tabular}

Tabela 2. Dados termoanaliticos das matérias-primas dos poliuretanos em atmosfera de $\mathrm{N}_{2}$.

\begin{tabular}{|c|c|c|c|c|c|c|c|c|c|c|c|c|}
\hline \multirow{2}{*}{ Amostra } & \multicolumn{3}{|c|}{ Primeira etapa } & \multicolumn{3}{|c|}{ Segunda etapa } & \multicolumn{3}{|c|}{ Terceira etapa } & \multicolumn{3}{|c|}{ Quarta etapa } \\
\hline & $\mathbf{T}^{\circ} \mathbf{C}$ & $\Delta \mathrm{m} \%$ & Pico $/{ }^{\circ} \mathrm{C}$ & $\mathbf{T}^{\circ} \mathbf{C}$ & $\Delta \mathrm{m} \%$ & Pico $/{ }^{\circ} \mathrm{C}$ & $\mathbf{T}^{\circ} \mathbf{C}$ & $\Delta \mathrm{m} \%$ & Pico/ $/{ }^{\circ} \mathrm{C}$ & $\mathbf{T}^{\circ} \mathrm{C}$ & $\Delta \mathrm{m} \%$ & Pico $/{ }^{\circ} \mathrm{C}$ \\
\hline OB & $70-144$ & 0,84 & - & $144-250$ & 3,34 & $185(\uparrow)$ & $250-492$ & 94,26 & $433(\downarrow)$ & - & - & - \\
\hline OBS & $51-81$ & 6,10 & $38,53(\downarrow)$ & $81-143$ & 15,92 & $\begin{array}{c}108,119, \\
120(\downarrow)\end{array}$ & $143-516$ & 72,35 & $\begin{array}{c}301,394, \\
459(\downarrow)\end{array}$ & $535-800$ & 1,97 & $726(\downarrow)$ \\
\hline Poliol & $52-181$ & 3,85 & - & $181-497$ & 93,72 & $381,446(\downarrow)$ & - & - & - & - & - & - \\
\hline PP & $139-354$ & 56,90 & $278(\downarrow)$ & $\begin{array}{l}354- \\
1000\end{array}$ & 19,01 & $542(\downarrow)$ & - & - & - & - & - & - \\
\hline
\end{tabular}

\subsubsection{TG-DSC do OBS}

As curvas TG-DSC do OBS em atmosfera de ar e $\mathrm{N}_{2}$ apresentaram perfis similares até $350{ }^{\circ} \mathrm{C}$, a distinção das curvas depois desta temperatura é decorrente da maior estabilidade térmica dos produtos intermediários oxidados formados em atmosfera de ar. As curvas TG do OBS até $138^{\circ} \mathrm{C}(\operatorname{ar})$ e $143^{\circ} \mathrm{C}\left(\mathrm{N}_{2}\right)\left(\Delta \mathrm{m}_{\mathrm{ar}}=33,41 \%\right.$ e $\left.\Delta \mathrm{m}_{\mathrm{N} 2}=31,85 \%\right)$, estão relacionadas desidratação do óleo (água de hidratação 
e constituição) provindas do processo de saponificação do OBB. Os resíduos formados em $650{ }^{\circ} \mathrm{C}$ (ar) e em $750{ }^{\circ} \mathrm{C}$ $\left(\mathrm{N}_{2}\right)$ foram, respectivamente, $3,33 \%(\mathrm{~m} / \mathrm{m})$ e $3,38 \%(\mathrm{~m} / \mathrm{m})$, estes resíduos são maiores do que os observados em $\mathrm{OBB}$, devido a inserção de $\mathrm{NaOH}$.

\subsubsection{TG-DSC do Poliol}

Apesar da similaridade nas curvas TG-DSC do poliol do óleo de mamona com o OBB, pode ser notado no terceiro evento de decomposição térmica, que a quantidade de calor liberado, obtido pela curva DSC, no OBB $\left(\Delta \mathrm{H}=1,01 \mathrm{~kJ} \mathrm{~g}^{-1}\right)$ foi significativamente maior que a observada na curva DSC do poliol $\left(\Delta \mathrm{H}=0,40 \mathrm{~kJ} \mathrm{~g}^{-1}\right)$, indicando característica intrínseca de cada matéria-prima estudada, e portanto podendo utilizar este parâmetro para distinguir aos dois óleos.

\subsubsection{TG-DSC do Pré-polímero}

A decomposição térmica do PP anidro, em ambas as atmosferas, ocorreram em duas etapas, com indicação de patamar entre $300-400{ }^{\circ} \mathrm{C}$. Em atmosfera de $\mathrm{N}_{2}$, houve a formação de resíduo carbonáceo $\left(\Delta \mathrm{m}_{\text {total }}=76,07 \%\right)$, e em atmosfera de ar ocorreu a degradação oxidativa da matéria orgânica, com perda de massa igual a 97,18\% do pré-polímero. O resíduo estável até $1000^{\circ} \mathrm{C}$, em atmosfera de ar, foi decorrente da presença de impureza de compostos inorgânicos do pré-polímero, podendo ser estimado a sua pureza em no máximo $97 \%(\mathrm{~m} / \mathrm{m})$.

\subsection{Análise Térmica do PU, PU-OBS 5,00\%, PU-OBS $15,00 \%$, PU-OBS $24,00 \%$ e PU-OBS $29,00 \%$}

\subsubsection{TG-DSC do PU}

As curvas TG-DSC da PU em atmosfera de ar seco e $\mathrm{N}_{2}$ apresentaram perfis distintos, devido as oxidações decorrentes da atmosfera em ar seco, ver Figuras 3 e 4 e as Tabelas 3 e 4 . A primeira perda de massa é relativo a saída de água de hidratação da PU, indicando que o material não é higroscópico. Teste qualitativo (visualização do resíduo) e a menor perda de massa $\left(\Delta \mathrm{m}-\mathrm{ar}=98,90 \%\right.$ e $\left.\Delta \mathrm{m}-\mathrm{N}_{2}=90,50 \%\right)$, sugere a formação de resíduo carbonáceo em atmosfera de $\mathrm{N}_{2}$. A massa residual em atmosfera de ar seco retrata a impureza da PU, provavelmente em função do material inorgânico presente nas matérias primas.

\subsubsection{TG-DSC do PU-OBS 5,00\%, PU-OBS 15,00\%, PU-OBS $24,00 \%$ e PU-OBS $29,00 \%$}

Os eventos observados nas curvas TG-DSC das quatro amostras de PUs contendo OBS (5,00\%, 15,00\%, 24,00\% e $29,00 \%$ ), em atmosfera de ar seco e $\mathrm{N}_{2}$, são mostrados nas Figuras 5 e 6 e Tabelas 3 e 4, sendo que os perfis das curvas apresentaram faixas de temperatura semelhantes. Apesar da estabilidade térmica da PU contendo OBS ser maior que a PU sem OBS, foi verificado que a estabilidade térmica das $\mathrm{PU}-\mathrm{OBS}$ diminuem à medida que a proporção em massa de OBS aumenta.

As curvas TG-DSC da espuma de PU sem OBS, apresentaram 2 picos exotérmicos em $320^{\circ} \mathrm{C}$ e $560{ }^{\circ} \mathrm{C}$, em atmosfera de ar seco, com perda de massa igual a $98,15 \%$ (Figura 3). As perdas de massa das PUs com OBS foram $96,16 \%$ (5,00\% OBS), 96,83\% (15,00\% OBS), 94,35\%
(24,00\% OBS) e $94,46(29,00 \%$ OBS $)$, sendo observados nas curvas DSCs 3 picos exotérmicos em aproximadamente $350{ }^{\circ} \mathrm{C}, 580{ }^{\circ} \mathrm{C}$ e $680^{\circ} \mathrm{C}$, ocasionados pela decomposição oxidativa dos materiais. Os picos exotérmicos em torno de $680^{\circ} \mathrm{C}$, observados nas curvas TG-DSC dos PUs-OBS, são relativos a decomposição oxidativa dos OBS nas PUs. A observação dos picos exotérmicos em $700{ }^{\circ} \mathrm{C}(5,00 \%$ OBS $)$, $681{ }^{\circ} \mathrm{C}(15,00 \%$ OBS $), 680{ }^{\circ} \mathrm{C}(24,00 \%$ OBS $)$ e $678{ }^{\circ} \mathrm{C}$ $(29,00 \%$ OBS $)$, sugere a interação dos OBS durante o processo de polimerização dos PUs. A quantidade de energia

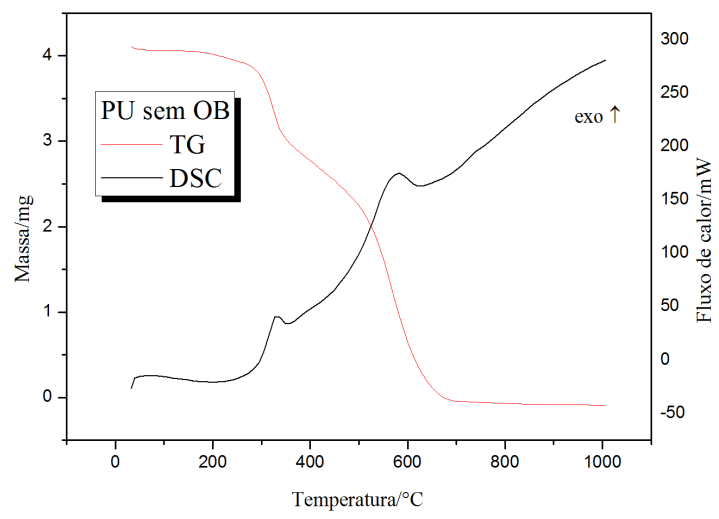

Figura 2. TG-DSC das matérias-primas dos poliuretanos em atmosfera de $\mathrm{N}_{2}$.

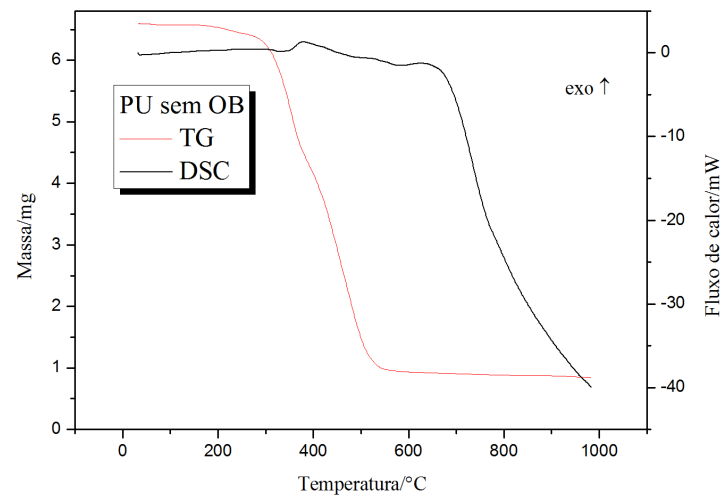

Figura 3. TG-DSC do poliuretano em atmosfera de ar.

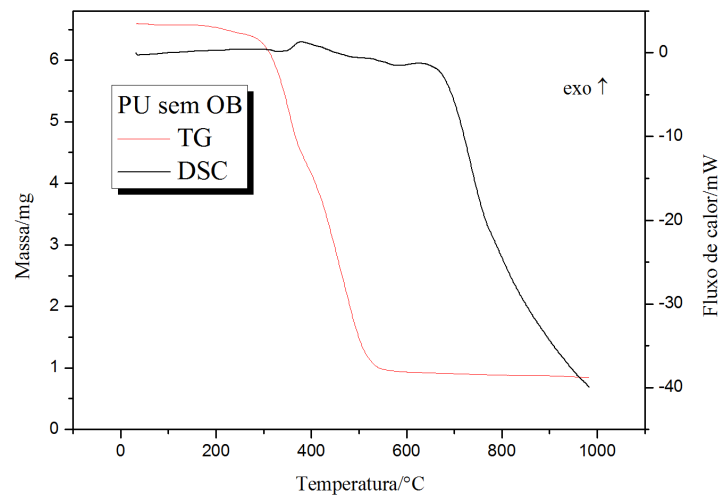

Figura 4. TG-DSC do poliuretano em atmosfera de $\mathrm{N}_{2}$. 
Almeida, E. L., Goulart, G. A. S., Claro, S., No., Chierice, G. O., \& Siqueira, A. B.

Tabela 3. Dados termoanalíticos dos PU's com diferentes concentrações de OBS em atmosfera de ar seco.

\begin{tabular}{|c|c|c|c|c|c|c|c|c|}
\hline \multirow{2}{*}{ Amostra } & \multicolumn{2}{|c|}{ Primeiro evento } & \multicolumn{2}{|c|}{ Segundo evento } & \multicolumn{2}{|c|}{ Terceiro evento } & \multicolumn{2}{|c|}{ Quarto evento } \\
\hline & $\mathbf{T}^{\circ} \mathbf{C}$ & $\Delta \mathrm{m} \%$ & $\mathbf{T}^{\circ} \mathbf{C}$ & $\Delta \mathrm{m} \%$ & $\mathbf{T}^{\circ} \mathbf{C}$ & $\Delta \mathrm{m} \%$ & $\mathbf{T}^{\circ} \mathbf{C}$ & $\Delta \mathrm{m} \%$ \\
\hline $\mathrm{PU}$ & $35-179$ & 0,75 & $179-356$ & 24,45 & $356-481$ & 14,53 & $481-695$ & 59,17 \\
\hline PU-OB-5\% & $33-108$ & 1,18 & $250-375$ & 24,19 & $375-681$ & 69,30 & $681-700$ & 2,67 \\
\hline PU-OB-15\% & $36-92$ & 0,73 & $200-364$ & 23,37 & $364-650$ & 65,50 & $650-680$ & 7,23 \\
\hline PU-OB-24\% & $33-84$ & 0,74 & $190-359$ & 24,06 & $359-643$ & 59,98 & $643-680$ & 10,31 \\
\hline PU-OB-29\% & $33-92$ & 0,86 & $180-363$ & 23,68 & $363-646$ & 60,90 & $646-693$ & 9,88 \\
\hline
\end{tabular}

Tabela 4. Dados termoanalíticos dos PU's com diferentes concentrações de OBS em atmosfera de $\mathrm{N}_{2}$.

\begin{tabular}{|c|c|c|c|c|c|c|c|c|}
\hline \multirow{2}{*}{ Amostra } & \multicolumn{2}{|c|}{ Primeiro evento } & \multicolumn{2}{|c|}{ Segundo evento } & \multicolumn{2}{|c|}{ Terceiro evento } & \multicolumn{2}{|c|}{ Quarto evento } \\
\hline & $\mathrm{T}^{\circ} \mathrm{C}$ & $\Delta \mathrm{m} \%$ & $\mathrm{~T}^{\circ} \mathrm{C}$ & $\Delta \mathrm{m} \%$ & $\mathbf{T}^{\circ} \mathrm{C}$ & $\Delta \mathrm{m} \%$ & $\mathrm{~T}^{\circ} \mathrm{C}$ & $\Delta \mathrm{m} \%$ \\
\hline PU & $34-173$ & 0,65 & $173-372$ & 29,27 & $372-540$ & 57,97 & $540-1000$ & 2,58 \\
\hline PU-OB-5\% & $33-108$ & 1,04 & $209-378$ & 38,30 & $378-553$ & 39,13 & $553-1000$ & 4,70 \\
\hline PU-OB-15\% & $34-112$ & 0,80 & $188-381$ & 38,62 & $381-551$ & 39,29 & $551-1000$ & 6,09 \\
\hline PU-OB-24\% & $33-84$ & 0,52 & $154-366$ & 33,87 & $366-541$ & 43,21 & $541-1000$ & 9,00 \\
\hline PU-OB-29\% & $29-88$ & 0,55 & $154-366$ & 33,43 & $366-542$ & 42,27 & $542-1000$ & 10,00 \\
\hline
\end{tabular}
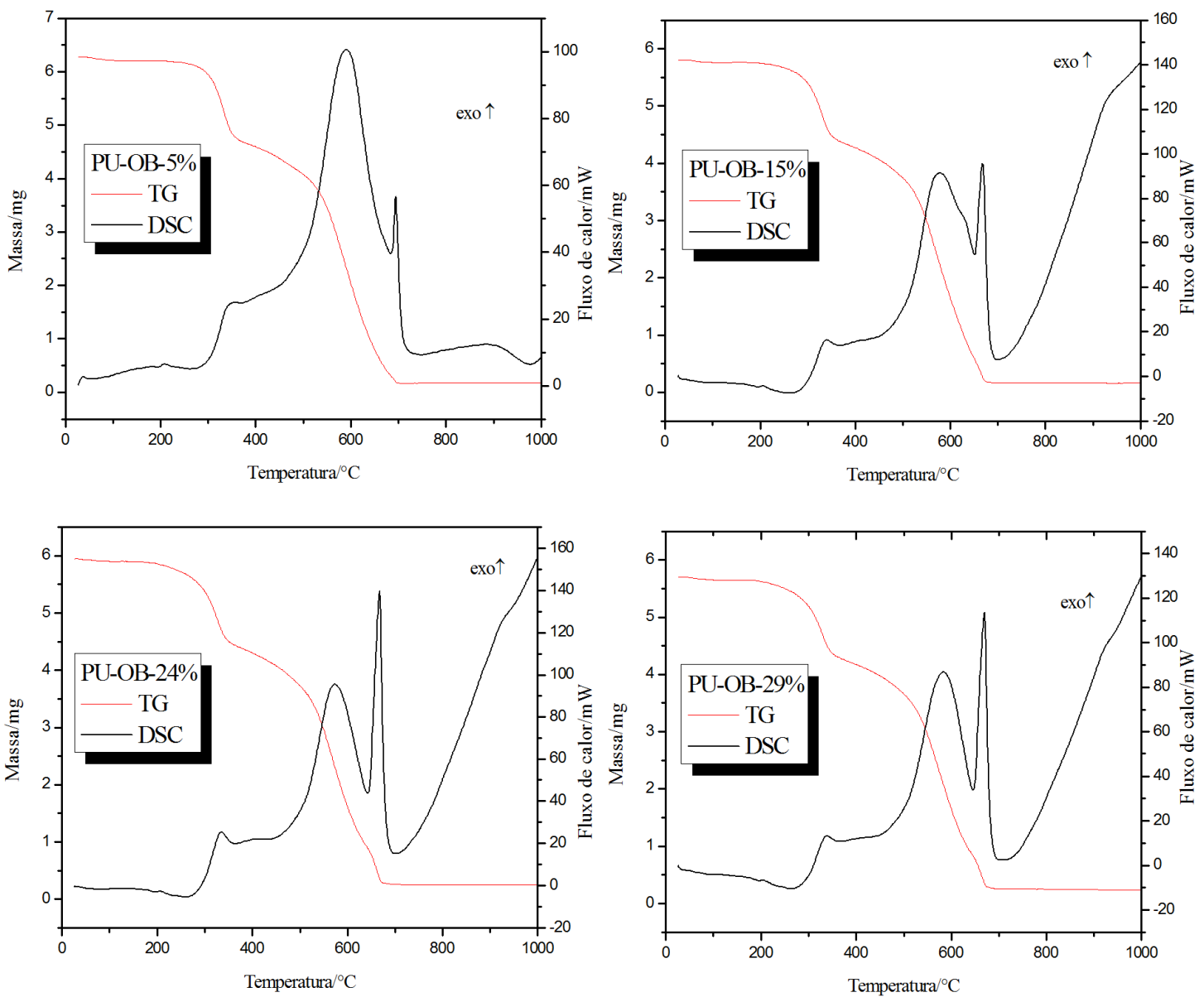

Figura 5. TG-DSC dos poliuretanos em atmosfera de ar.

calculado através das curvas DSC, $\left(\Delta \mathrm{H}_{50 \mathrm{OBS}}=92 \mathrm{~J} \mathrm{~g} \mathrm{~g}^{-1}\right.$; $\Delta \mathrm{H}_{15 \% \text { OBS }}=505 \mathrm{~J} \mathrm{~g} \mathrm{~g}^{-1} ; \Delta \mathrm{H}_{24 \% \mathrm{OBS}}=942 \mathrm{~J} \mathrm{~g}^{-1} ; \Delta \mathrm{H}_{5 \% \text { OBS }}=850 \mathrm{~J} \mathrm{~g}^{-1}$ ), assim como as perdas de massa observadas na quarta etapa de decomposição térmica da curva TG, indicam saturação do OBS nas reações de polimerização, quando utilizado $24 \%(\mathrm{~m} / \mathrm{m})$.
Nas amostras em atmosfera de $\mathrm{N}_{2}$ (Figura 6) foi observado um pico endotérmico em $360^{\circ} \mathrm{C}$ ocasionado pela decomposição térmica. $\mathrm{O}$ evento exotérmico com grande liberação de energia entre $600{ }^{\circ} \mathrm{C}$ e $820^{\circ} \mathrm{C}$, ocorreu devido a formação dos gases liberados na decomposição térmica, gerando reações oxidativas no sistema. Em função das 

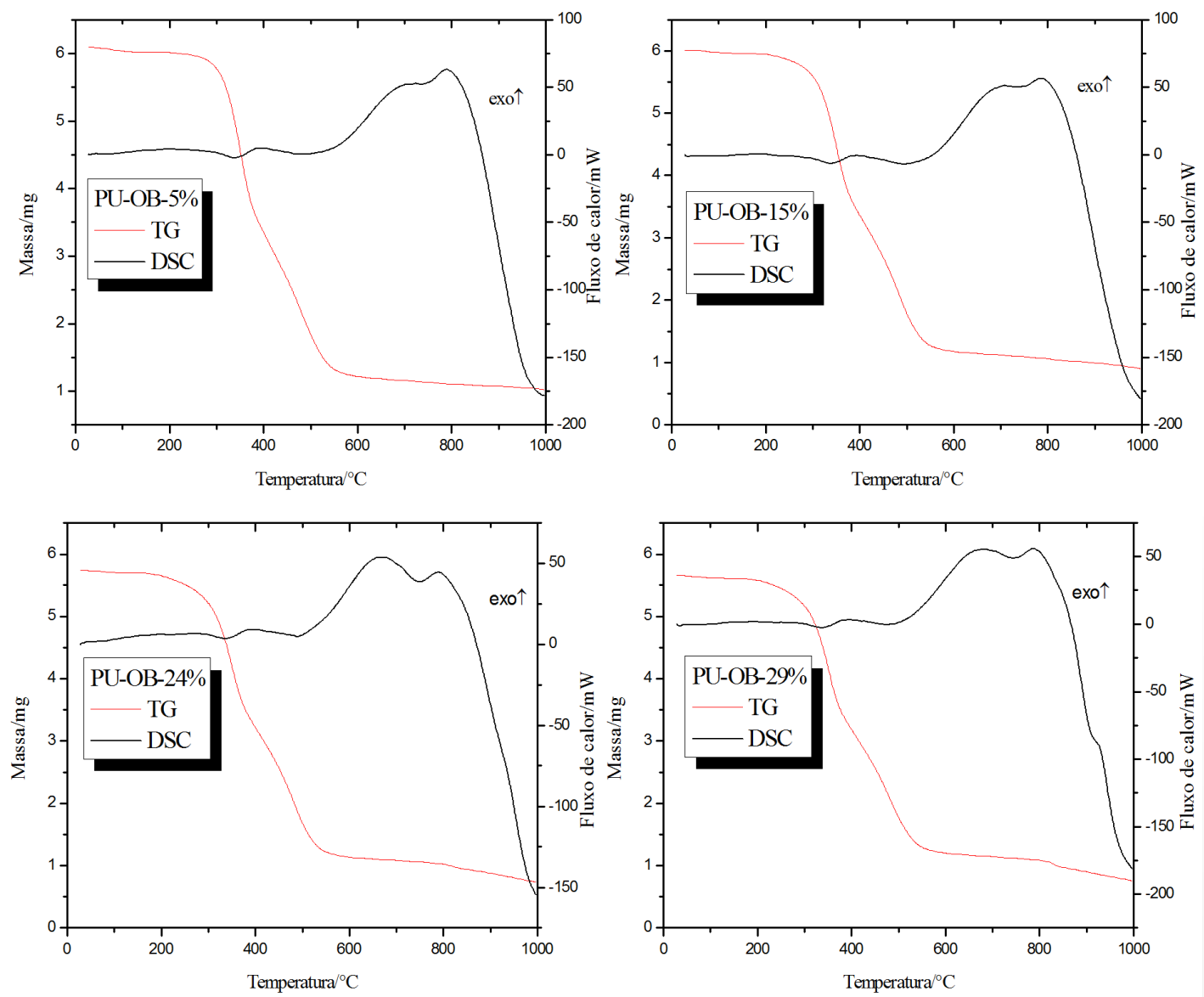

Figura 6. TG-DSC dos poliuretanos em atmosfera de $\mathrm{N}_{2}$.

reações de oxidação observadas nas curvas TG-DSC, não houve formação de resíduo carbonáceo.

\subsection{Espectroscopia de absorção na região do infravermelho}

As bandas observadas no espectro FTIR do OBB e OBS (Figura 7), foi verificado algumas semelhanças nas vibrações moleculares, com exceção da banda em $3380 \mathrm{~cm}^{-1}$, correspondente as interações de hidrogênio das hidroxilas, relativas ao processo de saponificação. Em comparação com os resultados de Drummond 2008, os picos que aparecem em $2922 \mathrm{~cm}^{-1}$ e $2853 \mathrm{~cm}^{-1}$ correspondem aos estiramentos da ligação $\mathrm{C}-\mathrm{H}$, atribuídos principalmente aos componentes lipídicos do óleo. Na região de $1744 \mathrm{~cm}^{-1}$ aparece um estiramento atribuído à ligação $\mathrm{C}=\mathrm{O}$, característico do grupo carbonilico do ácido graxo. A banda em $1160 \mathrm{~cm}^{-1} \mathrm{e} 721 \mathrm{~cm}^{-1}$ são atribuídos, respectivamente a ligação $\mathrm{C}-\mathrm{O}$ e $\mathrm{C}-\mathrm{H}$ dos ácidos graxos. Na Figura 8 está representado o espectro do poliol, onde pode ser observado as principais bandas em:

I) $3364 \mathrm{~cm}^{-1}$, atribuida a presença de hidroxila no óleo;

II) $1743 \mathrm{~cm}^{-1}$, referente ao grupo $\mathrm{C}=\mathrm{O}$;

III) $2925 \mathrm{~cm}^{-1}$ e $2845 \mathrm{~cm}^{-1}$, atribuídos, respectivamente, aos estiramentos simétrico e assimétrico do grupo $-\mathrm{CH}_{2}^{[2,4-9,11-14]}$.
O espectro de FTIR do pré-polímero (Figura 8), o mesmo material utilizado por Trovati et al., 2010 na caracterização do poliuretano de óleo de mamona, apresentou perfil semelhante ao encontrado neste trabalho, com banda característica do grupo isocianato em $2241 \mathrm{~cm}^{-1}$, e bandas indicando grupos uretanos polimerizados em $1718 \mathrm{~cm}^{-1}, 1608 \mathrm{~cm}^{-1}, 1577 \mathrm{~cm}^{-1} \mathrm{e}$ $1520 \mathrm{~cm}^{-1}$, bandas típicas de estiramentos $\mathrm{C}=\mathrm{O}$ e ligações N-H.

As principais bandas características de poliuretanos são as indicativas pela presença dos ésteres $(\mathrm{C}=\mathrm{O})$ do poliol, que estão em $1728 \mathrm{~cm}^{-1}$ e $1124 \mathrm{~cm}^{-1}$ e ainda em $814 \mathrm{~cm}^{-1}$ e $1598 \mathrm{~cm}^{-1}$ referentes aos isocianatos da estrutura do uretano ${ }^{[15,16]}$. A diminuição da intensidade da banda em $2250 \mathrm{~cm}^{-1}$ e a formação da banda característica de grupos uretanos polimerizados evidenciaram a formação do poliuretano, tais como bandas intensas na região de 3421-3447 e $1721 \mathrm{~cm}^{-1}$, referentes às ligações uretano, demonstrando consistência nas interações NCO-OH ${ }^{[17,18]}$.

Para todos os PUs-OBS (Figura 8) foi observado a banda em $1738 \mathrm{~cm}^{-1}$, característico do poliol referente ao grupo carbonila, sendo que ocorreu diminuição em sua intensidade relativa, à medida que a proporção de OBS aumenta, nas proporções de $15,00 \%, 24,00 \%$ e $29,00 \%$, indicando a inserção do OBS no PU. As bandas observadas em $3320 \mathrm{~cm}^{-1}$ são referentes aos grupos hidroxilas presentes no poliol e no OBS. 


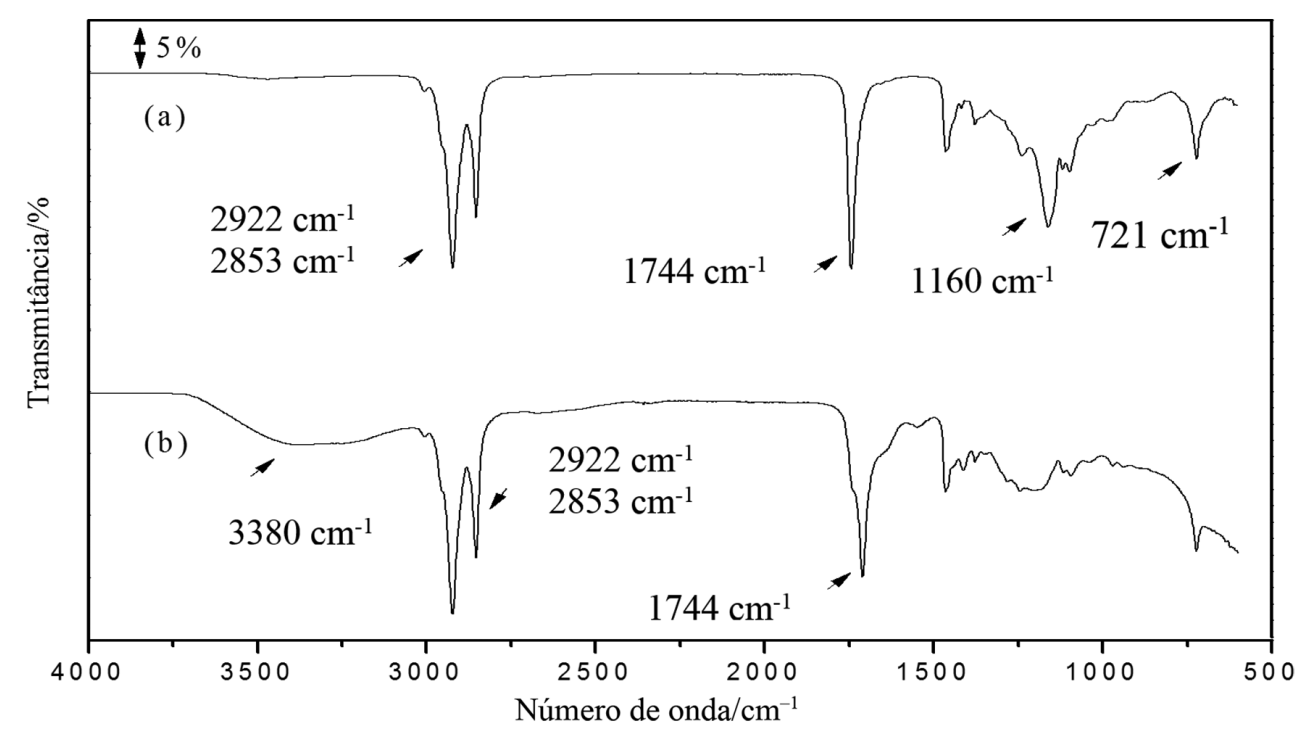

Figura 7. Espectros FTIR do (a) OBB e (b) OBS.

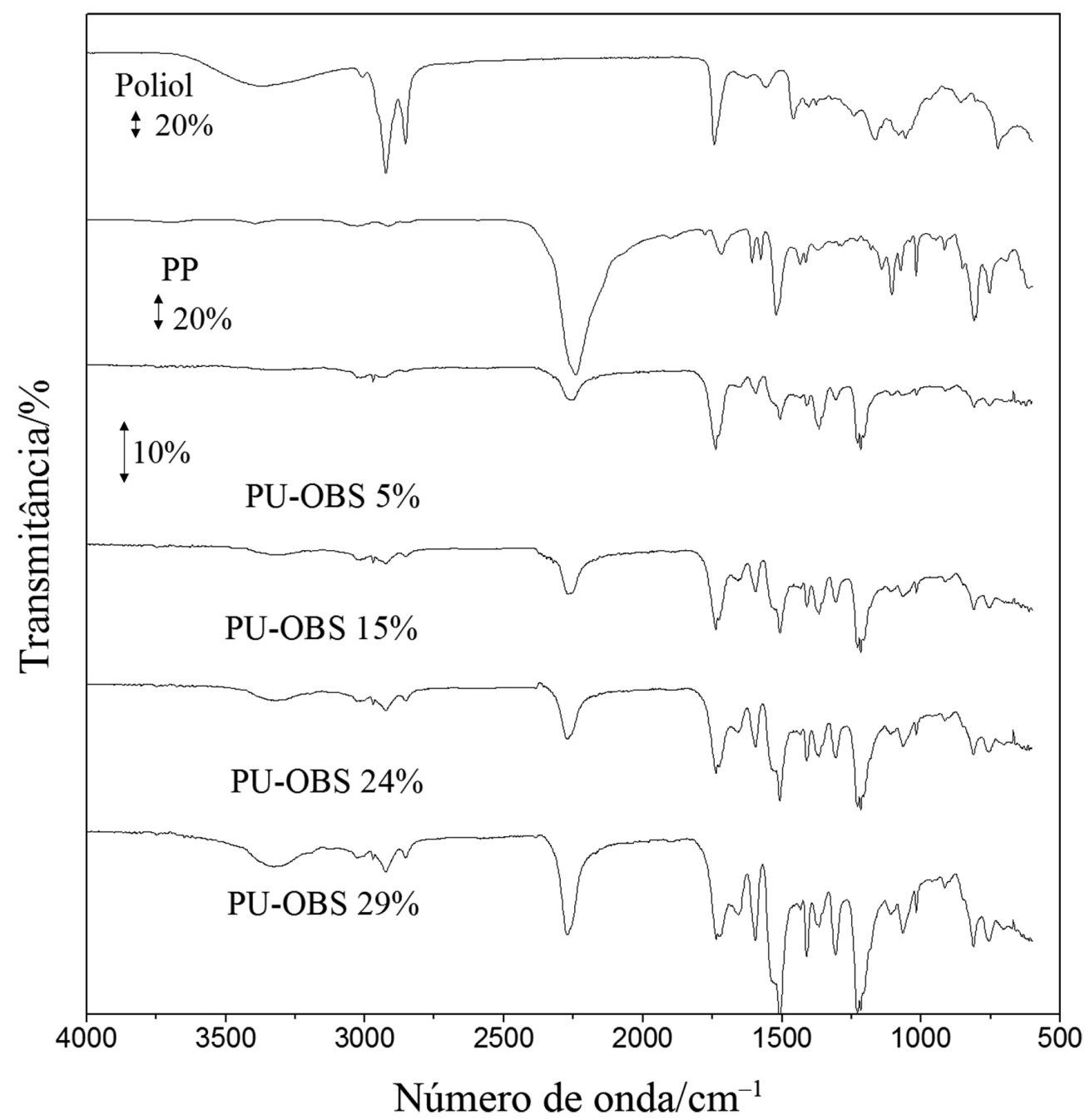

Figura 8. Espectros FTIR do: Polol, PP, PU-OBS 5\%, PU-OBS 15\%, PU-OBS 24\% e PU-OBS 29\%. 
Uma análise comparativa dos espectros das matérias primas, do PU e so PU-OBS sugerem ocorrência de polimerização com o OBS. Esta sugestão é devido ao deslocamento das bandas do espectro FTIR de $3354 \mathrm{~cm}^{-1}$ (OBS e poliol) para $3351 \mathrm{~cm}^{-1}$ (PU) e $3333 \mathrm{~cm}^{-1}$ (PUs-OBS-5\%/15\%/24\%/29\%), referentes aos estiramentos das hidroxilas. Os valores relativos de transmitância das PUs sugerem pouca alteração na quantidade de hidroxilas presentes na PU com as PUs-OBS, sendo que o valor observado no PU-OBS 29,00\% é similar ao observado no PU.

\subsection{Análise por FTIR dos gases liberados, acoplado ao TG-DSC}

Os produtos gasosos detectados por FTIR na decomposição térmica dos poliuretanos, com ou sem OBS, foram $\mathrm{CO}_{2}, \mathrm{H}_{2} \mathrm{O}$, etanol e CO, em ambas as atmosferas, ver Figura 9, com exceção do PU-OB-5\% que apresentou liberação somente de $\mathrm{CO}_{2}$, característica dos grupos isocianato, entre $50{ }^{\circ} \mathrm{C}$ até $370^{\circ} \mathrm{C}$. Nas análises dos gases do PU, PU-OBS 15\%, PU-OBS 24\%, e PU-OBS 29\%, ocorreu a liberação de $\mathrm{CO}_{2}$ na faixa de temperatura entre $30{ }^{\circ} \mathrm{C}$ até $410{ }^{\circ} \mathrm{C}, \mathrm{H}_{2} \mathrm{O}$ com formação de uma banda em $3500 \mathrm{~cm}^{-1}$, indicativo de hidroxila, banda em $1500 \mathrm{~cm}^{-1}$, típico de estiramento $\mathrm{C}=\mathrm{O}$ e ligações N-H dos uretanos.

Na atmosfera de ar seco, todos os poliuretanos mostraram comportamento semelhante na liberação de produtos gasosos, com exceção do PU-OB-5\% que não apresentou liberação de etanol na faixa de $230-300{ }^{\circ} \mathrm{C}$, os demais tratamentos apresentaram além do etanol, $\mathrm{CO}_{2}$ na faixa de temperatura entre $300-700{ }^{\circ} \mathrm{C}$. A saída de $\mathrm{H}_{2} \mathrm{O}$ pode ser observada em dois momentos, no início do processo de degradação entre $100-370{ }^{\circ} \mathrm{C}$ e nos momentos finais de $550-590{ }^{\circ} \mathrm{C}$. A saída de $\mathrm{CO}$ pode ser observado entre $390-630^{\circ} \mathrm{C}$.

\subsection{Indice de intumescimento}

A capacidade de adsorção de água nos PUs, com e sem OBS, foi avaliado pelo índice de intumescimento, ver Tabela 5. Somente o sistema PU-OBS 15,00\% apresentou ponto máximo de absorção de água aos 20 minutos $(42,80 \%)$, nos outros sistemas estudados houve aumento no percentual do índice de intumescimento com o aumento da massa do OBS, ao final das 24 horas de imersão. O aumento no índice de intumescimento pode ser explicado pelo aumento da hidrofilicidade do sistema, pois a difusão de água é

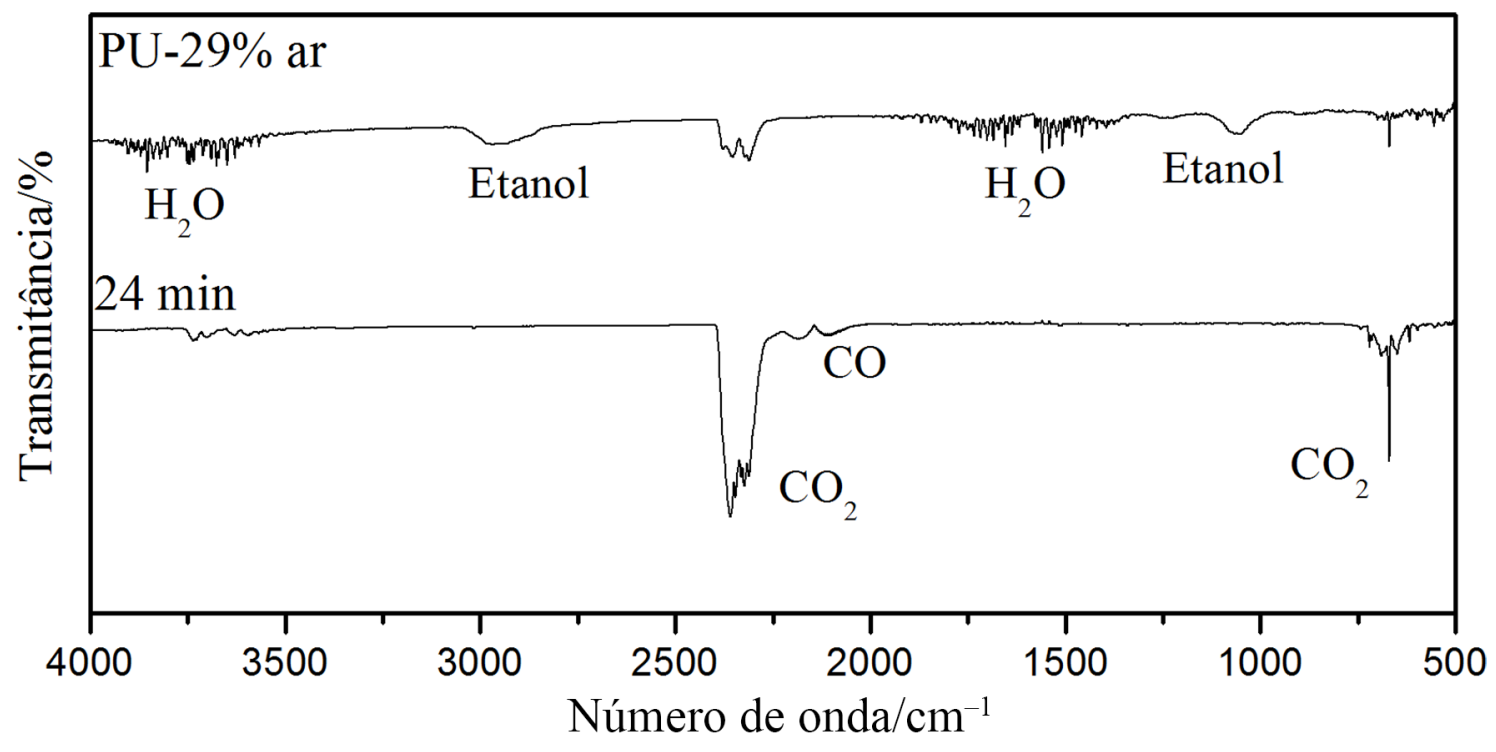

Figura 9. Espectros FTIR dos gases liberados do PU-OB 29\%, representativo a todos os PU-OBS.

Tabela 5. Índice de Intumescimento dos poliuretanos com diferentes concentrações do óleo de baru.

\begin{tabular}{ccccc}
\hline \multirow{2}{*}{ Tempo $($ min) } & \multicolumn{4}{c}{ Índice de Intumescimento \% } \\
\cline { 2 - 5 } & PU-OB-5,00\% & PU-OB-15,00\% & PU-OB-24,00\% & PU-OB-29,00\% \\
\hline 1 & 11,2 & 9,5 & 41,0 & 39,9 \\
3 & 15,8 & 15,7 & 45,3 & 23,5 \\
5 & 23,7 & 14,6 & 38,6 & 39,7 \\
7 & 27,9 & 20,4 & 30,4 & 41,3 \\
10 & 31,9 & 18,9 & 34,3 & 28,7 \\
15 & 32,9 & 11,8 & 44,8 & 22,7 \\
20 & 25,7 & 42,8 & 47,9 & 24,3 \\
30 & 25,1 & 32,2 & 46,5 & 21,2 \\
60 & 25,1 & 32,9 & 45,9 & 21,7 \\
1440 & 34,9 & 25,4 & 50,4 & 91,1 \\
\hline
\end{tabular}


determinada pela microestrutura do material e a afinidade dos componentes poliméricos pela água. .

Como o poliol favorece naturalmente a solubilidade em água devido a presença da hidroxila no carbono 12 da cadeia lipofílica, a adição do OBS no PU faz aumentar a absorção de água pelo poliuretano.

\section{Conclusão}

Através das curvas termoanalíticas, termogravimetriacalorimetria exploratória diferencial simultâneo (TG-DSC) podemos estudar as relações proporcionais das espumas formadas, estudar a estabilidade térmica e as etapas de decomposição térmica dos mesmos, assim como identificar a presença de impurezas nos materiais.

Apesar das curvas TG-DSC indicarem similaridades do OBB com o poliol, houve necessidade de saponificar o OBB para que o mesmo pudesse reagir na polimerização.

Os espectros de FTIR puderam confirmar a polimerização do PU, mesmo com a inserção de OBS, seugerindo inclusive grande similaridade nas propriedades dos mesmos.

$\mathrm{O}$ índice de intumescimento mostrou que o OBS aumenta a absorção de água pelo PU.

Tendo em vista a afinidade da PU-OBS por água, e sendo esta associação ocasionada pelas interações de hidrogênio água-OB, portanto, é provável que o sistema PU-OBS venha a interagir com metanol e etanol. Caso ocorra adsorção metanol e etanol, de forma seletiva, este sistema poderá ser utilizado na purificação do biodiesel.

\section{Agradecimentos}

A FAPEMAT e CNPq pelo suporte financeiro e Bolsa de mestrado concedido pela CAPES. A Engenheira de Alimentos Tatiane R. Silva por auxiliar na preparação do óleo de Baru bruto, utilizado neste trabalho.

\section{Referências}

1. Lopes, R. V. V. (2009). Poliuretanas obtidas a partir dos óleos de linhaça (Linun usitatissimum L.) e maracujá (Passiflora edulis Sims f. flavicarpa Degener): preparação e caracterização (Tese de Doutorado). Universidade de Brasília, Brasília.

2. Spontón, N., Casis, N., Mazo, P., Raud, B., Simonetta, A., Ríos, L., \& Estenoz, D. (2013). Biodegradation study by Pseudomonas sp. of flexible polyurethane foams derived from castor oil. International Biodeterioration \& Biodegradation, 85, 85-94. http://dx.doi.org/10.1016/j.ibiod.2013.05.019.

3. Corrêa, G. C., Naves, R. V., Rocha, M. R., Chaves, L. J., \& Borges, J. D. (2008). Determinações físicas em frutos e sementes de baru (Dipteryx alata Vog.), cajuzinho (Anacardium othonianum Rizz.) e pequi (Caryocar brasiliense Camb.), visando melhoramento genético. Jornal Biosciencia, 24(4), 42-47. Recuperado em 03 de Jun. de 2015, de http://www. seer.ufu.br.

4. Takemoto, E., Okada, I. A., Garbelotti, M. L., Tavares, M., \& Pimentel, S. A. (2001). Composição química da semente e do óleo de baru (Dipteryx alata Vog.) nativo do Município de Pirenópolis, Estado de Goiás. Revista do Instituto Adolfo Lutz, 60(2), 113-117. Recuperado em 03 de Jun. de 2015, de http://www.bioflorestal.com/baru.pdf

5. Sano, S. M., Ribeiro, J. F., \& Brito, M. A. (2004). Baru: biologia e uso. Planaltina: Embrapa Cerrados.
6. Vera, R., Soares, M., Naves, R. V., Souza, E. R. B., Fernandes, E. P., Caliari, M., \& Leandro, W. M. (2009). Características químicas de amêndoas de barueiros (dipteryx alata vog.) de ocorrência natural no cerrado do estado de Goiás, Brasil. Revista Brasileira de Fruticultura, 31(1), 118-112. http:// dx.doi.org/10.1590/S0100-29452009000100017.

7. Zhang, L., Jeon, H. K., Malsam, J., Herrington, R., \& Macosko, C. W. (2007). Substituting soybean oil-based polyol into polyurethane flexible foams. Polymer, 48(22), 6656-6667. http://dx.doi.org/10.1016/j.polymer.2007.09.016.

8. Ferrer, M. C. C., Babb, D., \& Ryan, A. J. (2008). Characterisation of polyurethane networks based on vegetable derived polyol. Polymer, 49(15), 3279-3287. http://dx.doi.org/10.1016/j. polymer.2008.05.017.

9. Kong, X., Liu, G., Qi, H., \& Curtis, J. M. (2013). Preparation and characterization of high-solid polyurethane coating systems based on vegetable oil derived polyols. Progress in Organic Coatings, 76(9), 1151-1160. http://dx.doi.org/10.1016/j. porgcoat.2013.03.019.

10. Pinheiro, A. P. S., Andrade, J. M., Melo, M. A. F., \& Araújo, D. M. (2006). Influência da hidroxila da cadeia lipofilica na formação e estabilidade da espuma do tensoativo ricinoleato de sódio proveniente do óleo de mamona. In Anais do $2^{\circ}$ Congresso Brasileiro de Mamona (pp. 1-6). Aracaju: Embrapa.

11. Soares, M. S. (2012). Síntese e caracterização de espumas de poliuretanos para imobilização de células integras e aplicação na sintese de biodiesel (Dissertação de Mestrado). Escola de Engenharia de Lorena, Universidade de São Paulo, São Paulo.

12. Pereira, V. A. Jr, Arruda, I. N. Q., \& Stefani, R. (2014). Active chitosan/PVA films with anthocyanins from Brassica oleraceae (Red Cabbage) as time-temperature Indicators for application in intelligent food packaging. Food Hydrocolloids, 2014, 1-9. http://dx.doi.org/10.1016/ j.foodhyd.2014.05.014.

13. Drummond, A. L. (2008). Compósitos poliméricos obtidos a partir do óleo de Baru: síntese e caracterização (Dissertação de Mestrado). Instituto de Química, Universidade de Brasília, Brasília.

14. Trovati, G., Sanches, E. A., Claro, S. No., Mascarenhas, Y. P., \& Chierice, G. O. (2010). Characterization of polyurethane resins by FTIR, TGA, and XRD. Journal of Applied Polymer Science, 115(1), 263-268. http://dx.doi.org/10.1002/app.31096.

15. Cangemi, J. M., Claro, S. No., Chierice, G. O., \& Santos, A. M. (2006). Study of the biodegradation of a polymer derived from castor oil by scanning electron microscopy, thermogravimetry and infrared spectroscopy. Polimeros: Ciência e Tecnologia, 16(2), 129-135. http://dx.doi.org/10.1590/ S0104-14282006000200013.

16. Pellizzi, E., Derieux, A. L., Lavédrine, B., \& Cheradame, H. (2013). Degradation of polyurethane ester foam artifacts: chemical properties, mechanical properties and comparison between accelerated and natural degradation. Polymer Degradation and Stability, 2013, 1-7. http://dx.doi.org/10.1016/j. polymdegradstab.2013.12.018.

17. Ismail, E. A., Motawie, A. M., \& Sadek, E. M. (2011). Synthesis and characterization of polyurethane coatings based on soybean oil-polyester polyols. Egyptian Journal of Petroleum, 20(2), 1-8. http://dx.doi.org/10.1016/j.ejpe.2011.06.009

18. Paiva, G. M. S., Borges, F. D. M., Batista, N. C., Lima, S. G. D., \& Matos, J. M. E. D. (2010). Sintese de poliuretana obtida do óleo de mamona (ricinus communis) na ausência de solventes. In Anais do $62^{\circ}$ Congresso Brasileiro para o Progresso da Ciência (pp. 1). Natal: SBPC. 